\title{
DrugEx v2: De Novo Design of Drug Molecule by Pareto- based Multi-Objective Reinforcement Learning in Polypharmacology
}

Xuhan Liu ${ }^{1}$, Kai Ye ${ }^{2}$, Herman W. T. van Vlijmen ${ }^{1,3}$, Michael T. M. Emmerich ${ }^{4}$, Adriaan P. IJzerman ${ }^{1}$, Gerard J. P. van Westen ${ }^{1, *}$

${ }^{1}$ Drug Discovery and Safety, Leiden Academic Centre for Drug Research, Einsteinweg 55, Leiden, The Netherlands

${ }^{2}$ School of electronics and information engineering, Xi'an Jiaotong University, 28 Xianning W Rd, Xi'an, China

${ }^{3}$ Janssen Pharmaceutica NV, Turnhoutseweg 30, B-2340, Beerse, Belgium

${ }^{4}$ Leiden Institute of Advanced Computer Science, Einsteinweg55, Leiden, The Netherlands

*To whom correspondence should be addressed: Gerard J. P. van Westen, Drug Discovery and Safety, Leiden Academic Centre for Drug Research, Einsteinweg 55, Leiden, The Netherlands. Tel: +31-71-527-3511. Email: gerard@lacdr.leidenuniv.nl.

Email Address of other authors: (1) Xuhan Liu: x.liu@lacdr.leidenuniv.nl; (2) Kai Ye: kaiye@xjtu.edu.cn; (3) Herman W. T. van Vlijmen: hvvlijme@its.jnj.com; (4) Michael T. M. Emmerich: m.t.m.emmerich@liacs.leidenuniv.nl; (5) Adriaan P. IJzerman: ijzerman@1acdr.leidenuniv.nl. 


\section{Abbreviations}

ARs

DL

MT-DNN

ECFP

EA

EDA

GPCRs

GRU

LSTM

QSAR

RBF

RMSE

ReLU

RF

RL

RNNs

SVM

t-SNE:
Adenosine Receptors

Deep Learning

Multi-Task Deep Neural Network

Extended Connectivity Fingerprint

Evolutionary Algorithm

Estimation of Distribution Algorithm

G Protein-coupled Receptors

Gated Recurrent Unit

Long Shot-Term Memory

Quantitative Structure-Activity Relationship

Radial Basis Function

Root Mean Square Error

Rectified Linear Unit

Random Forest

Reinforcement Learning

Recurrent Neural Networks

Support Vector Machine

t-distributed Stochastic Neighbor Embedding 


\section{Abstract}

In polypharmacology, ideal drugs are required to bind to multiple specific targets to enhance efficacy or to reduce resistance formation. Although deep learning has achieved breakthrough in drug discovery, most of its applications only focus on a single drug target to generate drug-like active molecules in spite of the reality that drug molecules often interact with more than one target which can have desired (polypharmacology) or undesired (toxicity) effects. In a previous study we proposed a new method named DrugEx that integrates an exploration strategy into RNN-based reinforcement learning to improve the diversity of the generated molecules. Here, we extended our DrugEx algorithm with multi-objective optimization to generate drug molecules towards more than one specific target (two adenosine receptors, $\mathrm{A}_{1} \mathrm{AR}$ and $\mathrm{A}_{2 \mathrm{~A}} \mathrm{AR}$, and the potassium ion channel hERG in this study). In our model, we applied an RNN as the agent and machine learning predictors as the environment, both of which were pre-trained in advance and then interplayed under the reinforcement learning framework. The concept of evolutionary algorithms was merged into our method such that crossover and mutation operations were implemented by the same deep learning model as the agent. During the training loop, the agent generates a batch of SMILESbased molecules. Subsequently scores for all objectives provided by the environment are used for constructing Pareto ranks of the generated molecules with non-dominated sorting and Tanimoto-based crowding distance algorithms. Here, we adopted GPU acceleration to speed up the process of Pareto optimization. The final reward of each molecule is calculated based on the Pareto ranking with the ranking selection algorithm. The agent is trained under the guidance of the reward to make sure it can generate more desired molecules after convergence of the training process. All in all we demonstrate generation of compounds with a diverse predicted selectivity profile toward multiple targets, offering the potential of high efficacy and lower toxicity. 


\section{Introduction}

The 'one drug, one target, one disease' paradigm, which has dominated the field of drug discovery for many years, has made great contributions to drug development and the understanding of their molecular mechanisms of action [1]. However, this strategy is encountering problems due to the intrinsic promiscuity of drug molecules, i.e. recent studies showed that one drug molecule could interact with six protein targets on average [2]. Side effects of drugs caused by binding to unexpected off-targets are one of the main reasons of clinical failure of drug candidates and even withdrawal of FDAapproved novel drugs $[3,4]$. Up to now, more than 500 drugs have been withdrawn from the market due to fatal toxicity [5]. Yet, disease often results from the perturbation of biological systems by multiple genetic and/or environmental factors, thus complex diseases are more likely to require treatment through modulating multiple targets simultaneously. Therefore, it is crucial to shift the drug discovery paradigm to "polypharmacology" for many complex diseases [6,7].

In polypharmacology, ideal drugs are required to bind to multiple specific targets to enhance efficacy or to reduce resistance formation (in which case multiple targets can be multiple mutants of a single target) [8]. It has been shown that partial inhibition of a small number of targets can be more efficient than the complete inhibition of a single target, especially for complex and multifactorial diseases [6,9]. In parallel, common structural and functional similarity of proteins results in drugs binding to off-targets; therefore we also demand drugs to have a high target selectivity to avoid binding to unwanted target proteins. For example, the adenosine receptors (ARs) are a class of rhodopsin-like G protein-coupled receptors (GPCRs) having adenosine as the endogenous ligand. Adenosine and ARs are ubiquitously distributed throughout the human tissues, and their interactions trigger a wide spectrum of physiological and pathological functions. There are four subtypes of $A R s$, including $A_{1}, A_{2 A}, A_{2 B}$ and $A_{3}$, each of which has a unique pharmacological profile, tissue distribution, and effector coupling [10,11]. The complexity of adenosine signaling and the widespread 
distribution of ARs have always given rise to challenges in developing target-specific drugs [12]. In addition to the similarity of the pharmacophores of some generic proteins (e.g. human Ether-à-go-go-Related Gene, hERG) should also be taken into consideration as they can be sensitive to binding exogenous ligands and cause side effects. hERG is the alpha subunit of a potassium ion channel [13] and has an inclination to interact with drug molecules because of its larger inner vestibule as the ligand binding pocket [14]. When hERG is inhibited this may cause long QT syndrome [15].

In addition to visual recognition, natural language processing and gaming, deep learning has been increasingly applied in drug discovery [16]. It does not only perform well in prediction models for virtual screening, but is also used to construct generative models for drug de novo design and/or drug optimization [17]. For example, our group implemented a fully-connected deep neural network (DNN) to construct a proteochemometric model (PCM) with all high quality ChEMBL data [18] for prediction of ligand bioactivity [19]. Its performance was shown to be better than other shallow machine learning methods. Moreover, we also developed a generative model with recurrent neural networks (RNNs), named DrugEx for SMILES-based de novo drug design [20]. It was shown that the generated molecules had large diversity and were similar to known ligands to some extent to make sure that reliable and diverse drug candidates can be designed.

Since the first version of DrugEx $(v l)$ demonstrated effectiveness for designing novel $\mathrm{A}_{2 \mathrm{~A}} \mathrm{AR}$ ligands, we began to extend this method for drug design toward multiple targets. In this study, we updated DrugEx to the second version (v2) through merging crossover and mutation operations, which were derived from evolutionary algorithms, into the reinforcement learning (RL) framework. In order to evaluate the performance of our additions we tested our method into both multi-target and target-specific cases. For the multi-target case, desired molecules should have a high affinity towards both $\mathrm{A}_{1} \mathrm{AR}$ and $\mathrm{A}_{2 \mathrm{~A}} \mathrm{AR}$. In the target-specific case, on the other hand, we required molecules to have only high affinity towards the $A_{2 A} A R$ but a low affinity to the $A_{1} A R$ for. In order to 
decrease toxicity and adverse events, molecules were additionally obliged to have a low affinity for hERG in both cases. It is worth noting that generated molecules should also be chemically diverse and have similar physico-chemical properties to known ligands. All python code for this study is freely available at http://github.com/XuhanLiu/DrugEx.

\section{Materials and Methods}

\section{Data Source}

Drug like molecules represented as SMILES format were downloaded from the ChEMBL database (version 26). After data preprocessing, including recombining charges, removing metals and small fragments, we collected 1.7 million molecules and named it the ChEMBL set, used for SMILES syntax learning. This data preprocessing step was implemented in RDKit [21]. Furthermore, 25,731 ligands were extracted from the ChEMBL database to construct the LIGAND set, which had bioactivity measurements towards the human $\mathrm{A}_{1} \mathrm{AR}, \mathrm{A}_{2 \mathrm{~A}} \mathrm{AR}$, and hERG. The LIGAND set was used for constructing prediction models for each target and fine-tuning the generative models. The number of ligands and bioactivities for these three targets in the LIGAND set is represented in Table 1. Duplicate items were removed and if multiple measurements for the same ligands existed, the average pChEMBL value $(\mathrm{pX}$, including pKi, pKd, pIC50, or pEC50) was calculated. To judge if a molecule is active or not, we defined the threshold of bioactivity as $\mathrm{pX}=6.5$. If the $\mathrm{pX}<6.5$, the compound was predicted as undesired (low affinity to the given target); otherwise, it was regarded as desired (having high affinity) [19].

\section{Prediction Model}

In order to predict the $\mathrm{pX}$ for each generated molecule for a given target, regression QSAR models were constructed with different machine learning algorithms. To increase the chemical diversity available for the QSAR model we included lower quality data without pChEMBL value, i.e. molecules that were labeled as "Not Active" 
or without a defined $\mathrm{pX}$ value. For these data points we defined a $\mathrm{pX}$ value of 3.99 (slightly smaller than 4.0) to eliminate the imbalance of the dataset and guarantee the model being able to predict the negative samples. During the training process, sample weights for low quality data were set as 0.1 , while the data with exact $\mathrm{pX}$ were set as 1.0. This allowed us to particularly incorporate the chemical diversity, while avoiding degradation of model quality. Descriptors used as input were ECFP6 fingerprints [22] with 2048 bits (2048 dimensions, or 2048D) calculated by the RDKit Morgan Fingerprint algorithm (using a three-bond radius). Moreover, the following 19D physico-chemical descriptors were used: molecular weight, $\log \mathrm{P}$, number of $\mathrm{H}$ bond acceptors and donors, number of rotatable bonds, number of amide bonds, number of bridge head atoms, number of hetero atoms, number of spiro atoms, number of heavy atoms, the fraction of SP3 hybridized carbon atoms, number of aliphatic rings, number of saturated rings, number of total rings, number of aromatic rings, number of heterocycles, number of valence electrons, polar surface area and Wildman-Crippen MR value. Hence, each molecule in the dataset was transformed into a 2067D vector. Before being input into the model, the value of input vectors were normalized to the range of $[0,1]$ by the MinMax method. Model output value is the probability whether a given chemical compound was active based on this vector.

Table 1: The number of ligands and bioactivities for each of the human protein targets $A_{1} A R$, $A_{2 A} A R$ and hERG in the LIGAND set.

\begin{tabular}{|c|c|c|c|}
\hline & $\mathbf{A}_{1} \mathbf{A R}$ & $\mathbf{A}_{2 \mathrm{~A}} \mathrm{AR}$ & hERG \\
\hline Total Ligands & 7700 & 8406 & 16733 \\
\hline Bioactivities & 13100 & 12129 & 22156 \\
\hline $\begin{array}{l}\text { Active Ligands } \\
\qquad(\mathbf{p X}>=6.5)\end{array}$ & 1990 & 2511 & 924 \\
\hline $\begin{array}{c}\text { Inactive Ligands } \\
\qquad(\mathbf{p X}<6.5)\end{array}$ & 1859 & 1709 & 6438 \\
\hline $\begin{array}{l}\text { Inactive Ligands } \\
\text { (No pX) }\end{array}$ & 1764 & 1993 & 1275 \\
\hline Other Ligands & 2087 & 4704 & 8906 \\
\hline
\end{tabular}


167 Four algorithms were benchmarked for QSAR model construction, Random Forest

168 (RF), Support Vector Machine (SVM), Partial Least Squares regression (PLS), and 169 Multi-task Deep Neural Network (MT-DNN). RF, SVM and PLS models were 170 implemented through Scikit-Learn [23], and the MT-DNN model through PyTorch [24].

171 In the RF, the number of trees was set as 1000 and split criterion was "gini". In the 172 SVM, a radial basis function (RBF) kernel was used and the parameter space of $\mathrm{C}$ and $173 \gamma$ were set as $\left[2^{-5}, 2^{15}\right]$ and $\left[2^{-15}, 2^{5}\right]$, respectively. In the MT-DNN, the architecture 174 contained three hidden layers activated by a rectified linear unit (ReLU) between input 175 and output layers, and the number of neurons were 2048, 4000, 2000, 1000 and 3 in 176 these subsequent layers. The training process consisted of 100 epochs with $20 \%$ of 177 hidden neurons randomly dropped out between each layer. The mean squared error was 178 used to construct the loss function and was optimized by the Adam algorithm [25] with 179 a learning rate of $10^{-3}$.

\section{Generative Model}

182 As in DrugEx vl, we organized the vocabulary for the SMILES construction. Each 183 SMILES-format molecule in the ChEMBL and LIGAND sets was split into a series of 184 tokens. Then all tokens existing in this dataset were collected to construct the SMILES vocabulary. The final vocabulary contained 85 tokens (Table S1) which were selected and arranged sequentially into valid SMILES sequences through correct grammar.

188 The RNN model constructed for sequence generation contained six layers: one input layer, one embedding layer, three recurrent layers and one output layer. After being represented by a sequence of tokens, molecules can be received as categorical features by the input layer. In the embedding layer, vocabulary size, and embedding dimension were set to 85 and 128 , meaning each token could be transformed into a 128

193 dimensional vector. For a recurrent layer, the long-short term memory (LSTM) was used as recurrent cell with 512 hidden neurons instead of the gated recurrent unit (GRU)

195 [26] which was employed only in DrugEx $v 1$. The output at each position was the 
196

197

198

199

200

201

202

203

204

205

206

207

208

209

210

211

212

213

214

215

216

217

218

219

probability that determined which token in the vocabulary would be chosen to grow the SMILES string.

During the training process we put a start token $(\mathrm{GO})$ at the beginning of a batch of data as input and an end token (END) at the end of the same batch of data as output. This ensures that our generative network could choose correct tokens each time based on the sequence it had generated previously. A negative log likelihood function was used to construct the loss function to guarantee that the token in the output sequence had the largest probability to be chosen after being trained. In order to optimize the parameters of the model, the Adam algorithm [25] was used for the optimization of the loss function. Here, the learning rate was set at $10^{-3}$, the batch size was 512, and training steps were set to 1000 epochs.

\section{Reinforcement Learning}

SMILES sequence construction under the RL framework can be viewed as a series of decision-making steps (Fig. 1). The generator $(G)$ and the predictors $(Q)$ are regarded as the policy and reward function, respectively. In this study we use multi-objective optimization (MOO), and each objective is a requirement to be achieved maximally for each scenario, albeit with differences in desirability. Our aim was defined by the following problem statement:

$$
\text { maximize } R_{1}, \quad \operatorname{maximize} R_{2}, \quad \ldots, \quad \operatorname{maximize} R_{n}
$$

Here, $n$ equals the number of objectives ( $n=3$ in this study), and $R_{i}$, the score for each objective $i$, was calculated as follows:

$$
R_{i}=\left\{\begin{aligned}
\operatorname{minmax}\left(p X_{i}\right), & \text { if high affinity required } \\
1-\operatorname{minmax}\left(p X_{i}\right), & \text { if low affinity required } \\
0, & \text { if SMILES invalid }
\end{aligned}\right.
$$




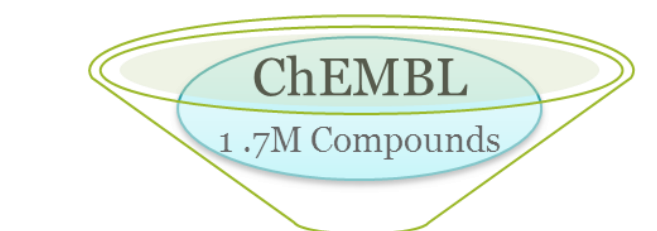

Pre-training

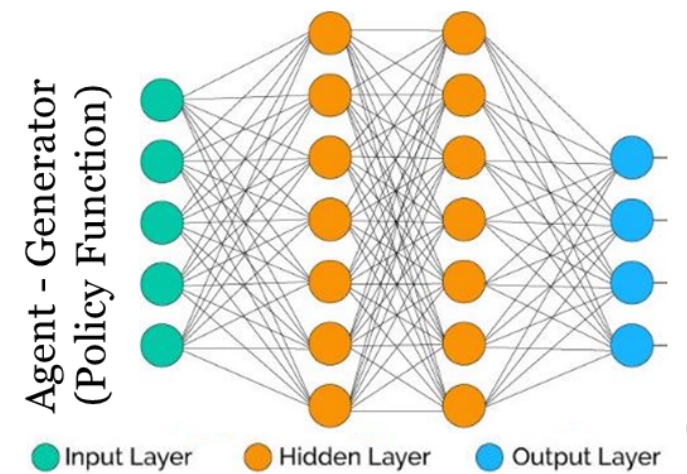

Input Layer Hidden Layer Output Layer

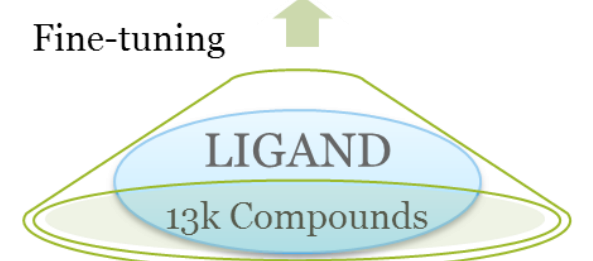

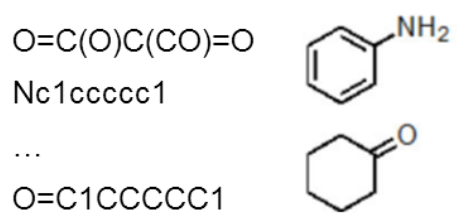

$\mathrm{O}=\mathrm{C} 1 \mathrm{Ccccc} 1$

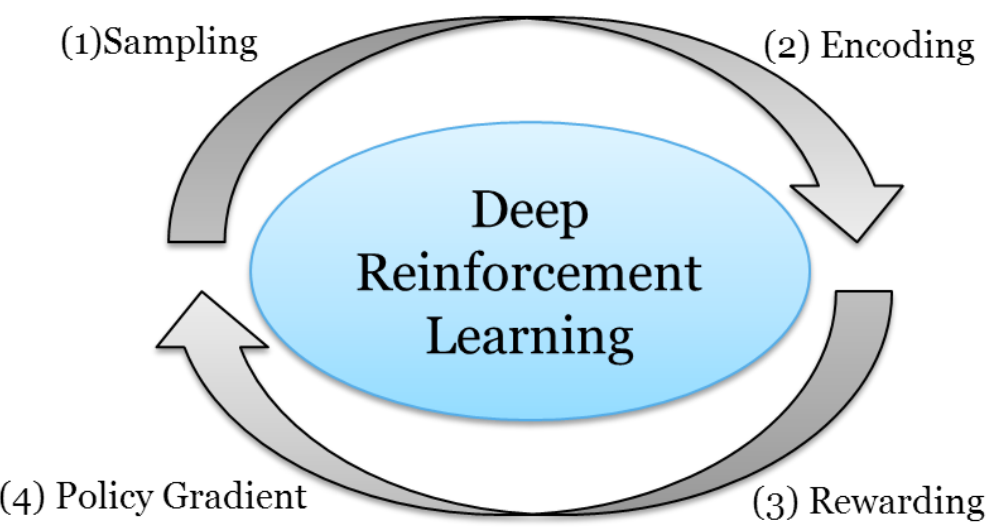

$\begin{array}{ll}\text { SMILES } & \text { Reward } \\ \mathrm{O}=\mathrm{C}(\mathrm{O}) \mathrm{C}(\mathrm{CO})=\mathrm{O} & 0.85 \\ \mathrm{Nc} 1 \mathrm{ccccc} 1 & 0.23 \\ \ldots & \ldots \\ \mathrm{O}=\mathrm{C} 1 \mathrm{ccccc} 1 & 0.38\end{array}$

$\begin{array}{lllllllllll}0 & 1 & 0 & 0 & 1 & 0 & 0\end{array}$

$\begin{array}{lllllll}1 & 0 & 1 & 1 & 0 & 0 & 1\end{array}$

$\begin{array}{llllllll}0 & 1 & 1 & 0 & 1 & 0 & 1\end{array}$

$\begin{array}{llllllllllllllll}0 & 0 & 1 & 1 & 1 & 1 & 0\end{array}$

(2) Encoding

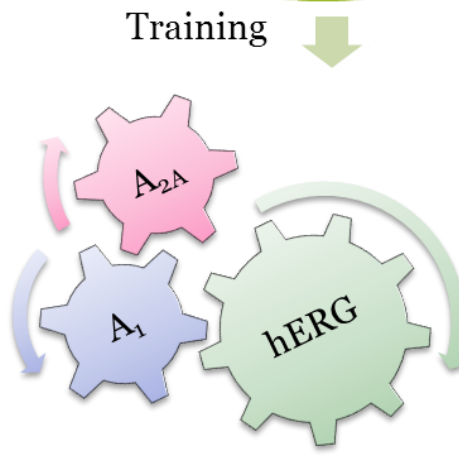

Predicting

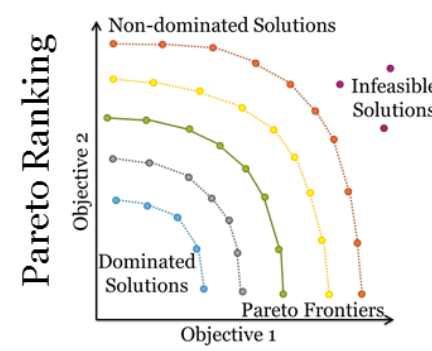

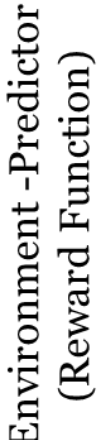

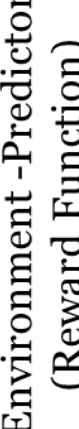

$\begin{array}{llll}\text { Mol } & \mathbf{A}_{\mathbf{1}} & \mathbf{A}_{\mathbf{2 A}} & \text { ERG } \\ \text { Mol 1 } & 0.81 & 0.90 & 0.78 \\ \text { Mol 2 } & 0.32 & 0.15 & 0.44 \\ \ldots & \ldots & \ldots & \ldots \\ \text { Mol n } & 0.38 & 0.43 & 0.55\end{array}$

Fig. 1: The workflow of the training process of our deep learning-based molecule generator DrugEx 2 utilizing reinforcement learning. After the generator has been pre-trained/fine-tuned, (1) a batch of SMILES are generated by sampling tokens step by step based on the probability calculated by the generator; (2) These valid SMILES are parsed to be molecules and encoded into descriptors to get the predicted pXs with well-trained predictors; (3) The predicted pXs are transformed into a single value as the reward for each molecule based on Pareto optimization; (4) These SMILES sequences and their rewards are sent back to the generator for training with policy gradient methods. These four steps constitute the training loop of reinforcement learning. 
22

here the $p X_{i}$ (the range from 3.0 to 10.0) was the prediction score given by each predictor for the $i^{t h}$ target, which was normalized to the interval $[0,1]$ as the reward score. If having no or low affinity for a target was required (off-target) this score would be subtracted from 1 (inverting it).

In order to evaluate the performance of the generators, three coefficients are calculated with the generated molecules, including validity, desirability, and uniqueness which are defined as:

$$
\begin{gathered}
\text { Validity }=\frac{N_{\text {valid }}}{N_{\text {total }}} \\
\text { Desirability }=\frac{N_{\text {desired }}}{N_{\text {total }}}
\end{gathered}
$$

$$
\text { Uniqueness }=\frac{N_{\text {unique }}}{N_{\text {total }}}
$$

where $N_{\text {total }}$ is the total number of molecules, $N_{\text {valid }}$ is the number of the molecules parsed by the valid SMILES sequences, $N_{\text {unique }}$ is the number of molecules which are different from others in the dataset, and $N_{\text {desired }}$ is the number of desired molecules. Here, we determine if generated molecules are desired based on the reward $R_{i}$ if all of them are larger than the threshold $(0.5$ by default when $\mathrm{pX}=6.5)$. In addition, we calculated SA score (from 1 to 10) for each molecule to measure the synthesizability of which larger value means more difficult to be synthesized. And we also computed QED (from 0 to 1) score to evaluate the drug-likeness of which larger value means more drug-like for each molecule. The calculation of both SA and QED scores were implemented by RDKit.

To orchestrate and combine these different objectives, we compared two different reward schemes: the Pareto front (PF) scheme and the weighted sum (WS) scheme. These were defined as follows:

(a) Weighted sum (WS) scheme: the weight for each function is not fixed but dynamic, and depends on the desired ratio for each objective, which is defined as:

$$
\mathrm{r}_{i}=\frac{N_{i}^{S}}{N_{i}^{l}}
$$


here for objective $i$ the $N_{i}^{s}$ and $N_{i}^{l}$ are the number of generated molecules which have a score smaller or larger than the threshold. Moreover, the weight is normalized ratio defined as:

$$
w_{i}=\frac{r_{i}}{\sum_{k=1}^{M} r_{k}}
$$

and the final reward $R^{*}$ was calculated by

$$
R^{*}=\sum_{i=1}^{n} w_{i} R_{i},
$$

(b) Pareto front (PF) scheme: operates on the desirability score, which is defined as

$$
\mathrm{D}_{i}=\left\{\begin{array}{c}
1, \quad \text { if } R_{i}>t_{i} \\
R_{i} / t_{i}, \quad \text { if } R_{i} \leq t_{i}
\end{array}\right.
$$

where $t_{i}$ is the threshold of the $i^{\text {th }}$ objective, and we set all of objectives had the same threshold as 0.5 as stated in the methods. Given two solutions $m_{1}$ and $m_{2}$ with their scores $\left(x_{1}, x_{2}, \ldots, x_{n}\right)$ and $\left(y_{1}, y_{2}, \ldots, y_{n}\right)$, then $m_{1}$ is said to Pareto dominate $m_{2}$ if and only if:

$$
\forall \mathrm{j} \in\{1, \ldots, \mathrm{n}\}: x_{j} \geq y_{j} \text { and } \exists \mathrm{j} \in\{1, \ldots, \mathrm{n}\}: x_{j}>y_{j}
$$

otherwise, $m_{1}$ and $m_{2}$ are non-dominated with each other. After the dominance between all pair of solutions being determined, the non-dominated scoring algorithm [27] is exploited to obtain a rank of Pareto frontiers which consist of a set of solutions. The solutions in the top frontier are dominated by the other solutions in the bottom frontier, but the solutions in the same frontier are non-dominated with each other [28]. In order to speed up the non-dominated sorting algorithm, we employed PyTorch to implement this procedure with GPU acceleration. After obtaining the frontiers ranking from dominated solutions to dominant solutions, the molecules were ranked based on the average of Tanimoto-distance instead of crowding distance with other molecules in the same frontier, and molecules with smaller distances were ranked on the top. The final reward $R^{*}$ is defined as:

$$
\mathrm{R}_{i}^{*}=\left\{\begin{array}{c}
0.5+\frac{k-N_{\text {undesired }}}{2 N_{\text {desired }}}, \quad \text { if desired } \\
\frac{k}{2 N_{\text {undesired }}}, \quad \text { if undesired }
\end{array}\right.
$$

here the parameter $k$ is the index of the solution in the Pareto rank, and rewards of 
undesired and desired solutions will be evenly distributed in $(0,0.5]$ and $(0.5,0.1]$, respectively.

During the generation process, for each step, $G$ determines the probability of each token from the vocabulary to be chosen based on the generated sequence in previous steps. Its parameters are updated by employing a policy gradient based on the expected end reward received from the predictor. The objective function is designated as follows:

$$
J(\theta)=\mathbb{E}\left[R^{*}\left(y_{1: T}\right) \mid \theta\right]=\sum_{t=1}^{T} \log G\left(y_{t} \mid y_{1: t-1}\right) \cdot R^{*}\left(y_{1: T}\right)
$$

By maximizing this function, the parameters $\theta$ in $G$ can be optimized to ensure that $G$ can construct desired SMILES sequences which can obtain the highest reward scores judged by all the $Q s$.

\section{Algorithm extrapolation}

Evolutionary algorithms (EAs) are common methods used in drug discovery [29]. For example, Molecule Evoluator is one of EAs, with mutation and crossover operations based on SMILES representation [30] for drug de novo design. In addition, some groups also proposed other variations of EAs [31], e.g., estimation of distribution algorithm (EDA) which is a model-based method and replaces the mutation and crossover operations with probability distribution estimation and sampling of new individuals (Fig. 2) [32]. Similar to EDA, DrugEx is a model-based method too, in which the deep learning model was employed to estimate the probability distribution of sequential decision making. However, we use a DL method to define model-based mutation and crossover operations. Moreover, we employed an RL method to replace the sample selection step for the update of model or population in EDA or EA, respectively. 

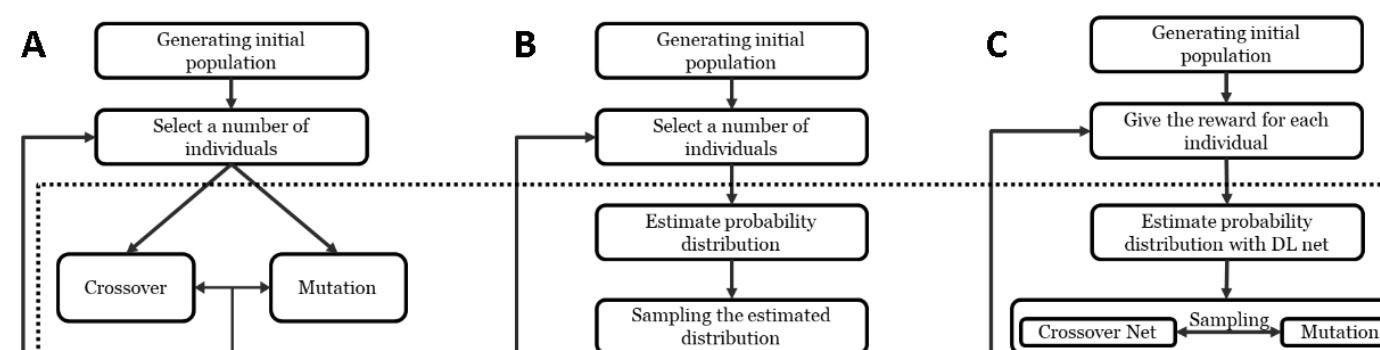

Fig. 2: Flowchart comparison of evolutionary algorithm (A), estimation of distribution algorithm (B) and our proposed method (C).

\section{Exploration Strategy}

311 In our previous study, we had implemented the exploration strategy through importing 312 a fixed exploration net to enlarge the diversity of the generated molecules during the 313 training loops. In this study, we continued to extend the methods of this exploration 314 strategy, which resemble the crossover and mutation operations from evolutionary algorithms (EAs). Here, besides the agent net $\left(G_{A}\right)$, we also defined exploration strategy with two other DL models: crossover net $\left(G_{C}\right)$ and mutation net $\left(G_{M}\right)$, which have the same RNN architecture (Fig. 3). Before the training process, they were initialized by a pre-trained or fine-tuned model. The $G_{M}$ was the basic strategy employed in the previous version and its parameters were fixed and not updated during the whole training process. The $G_{C}$ implemented in this work was an extended strategy whose parameters were updated iteratively based on the $\mathrm{G}_{A}$. During the training process, each SMILES sequence was generated through combining these three RNNs: for each step, a random number from 0 to 1 is generated. If it is larger than the mutation rate $(\varepsilon)$, the probability for token sampling is controlled by the combination of $G_{A}$ and $G_{C}$, otherwise, it is determined by $G_{M}$. For each training loop, only the parameters in $G_{A}$ were updated instantly based on the gradient of the RL objective function. An iteration was defined as the period of epochs after the desirability score of molecules generated by $G_{A}$ did not

328 increase. Subsequently the parameters of $G_{C}$ were updated with $G_{A}$ directly and the 
training process continued for the next iteration. The training process would continue

till the percentage of desired molecules in the current iteration was not better than in

the previous iterations.

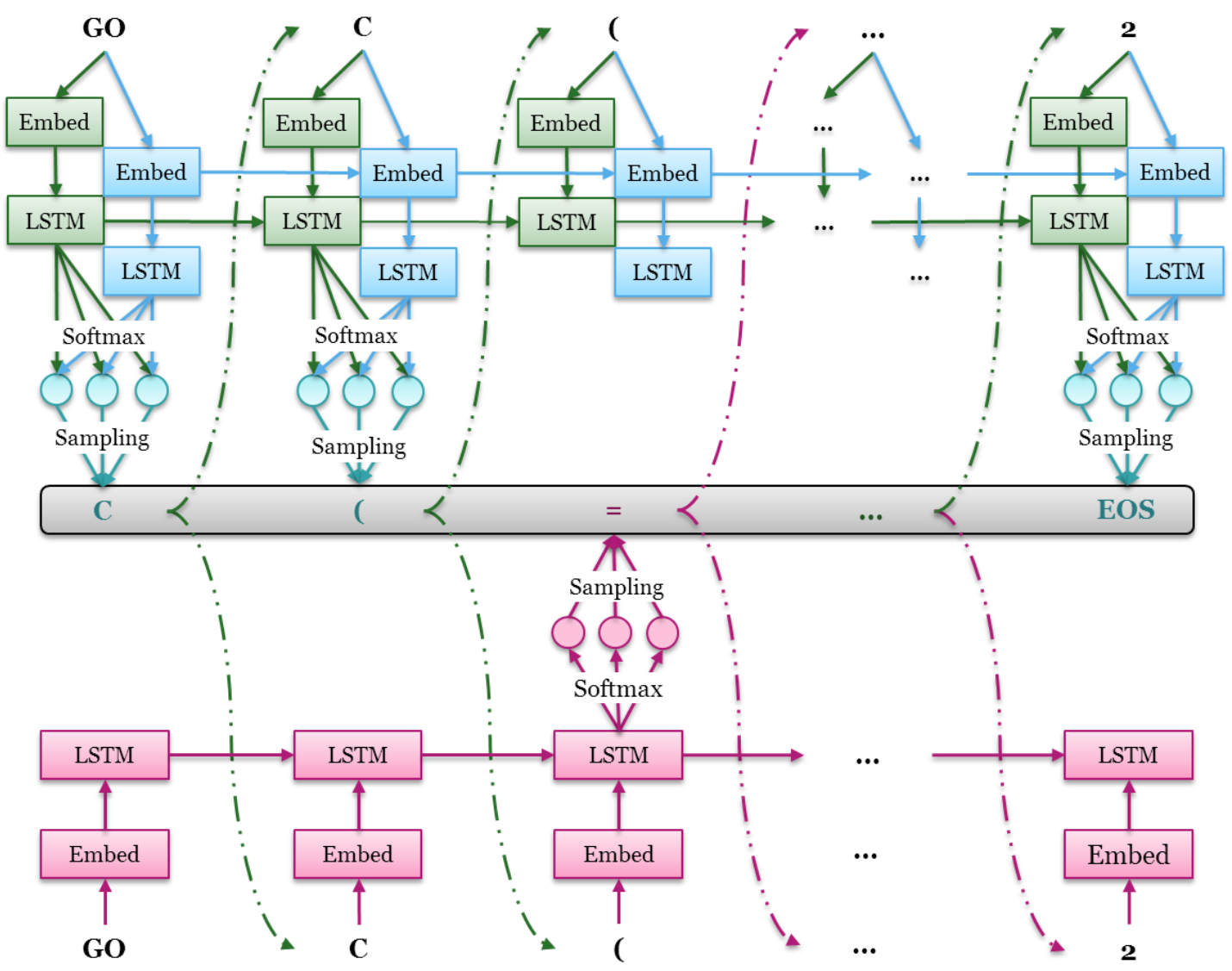

Fig. 3: The mechanism of updated exploration strategy, including agent net $G_{A}$, mutation net

$\boldsymbol{G}_{M}$ (red) and crossover net $\boldsymbol{G}_{C}$ (blue). In the training loop, $G_{M}$ is fixed, $G c$ is updated iteratively and $G_{A}$ is trained at each epoch. For each position, a random number from 0 to 1 is generated. If it is larger than the mutation rate $(\varepsilon)$, the probability for token sampling is controlled by the combination of $G_{A}$ and $G_{C}$, otherwise, it is determined by $G_{M}$.

\section{Molecular Diversity}

341 To measure molecular diversity, we adopted the metric proposed by Solow and Polasky in 1994 to estimate the diversity of a biological population in an eco-system [33]. It has

343 been shown to be an effective method to measure the diversity of drug molecules [34].

344 The formula to calculate diversity was redefined to normalize the range of values from $345[1, \mathrm{~m}]$ to $(0, \mathrm{~m}]$ as follows:

$$
I(A)=\frac{1}{|A|} \boldsymbol{e}^{\top} F(\boldsymbol{s})^{-1} \boldsymbol{e}
$$


where $A$ is a set of drug molecules with a size of $|A|$ equal to $m, \boldsymbol{e}$ is an $m$-vector of 1 's and $\left.F(s)=\left[f\left(d_{i j}\right)\right)\right]$ is a non-singular $m \times m$ distance matrix, in which $f\left(d_{i j}\right)$ stands for the distance function of each pair of molecule provided as follows:

$$
f(d)=e^{-\theta d_{i j}}
$$

here we defined the distance $d_{i j}$ of molecules $s_{i}$ and $s_{j}$ by using the Tanimoto-distance with ECFP6 fingerprints as follows:

$$
d_{i j}=d\left(s_{i}, s_{j}\right)=1-\frac{\left|s_{i} \cap s_{j}\right|}{\left|s_{i} \cup s_{j}\right|},
$$

where $\left|s_{i} \cap s_{j}\right|$ represents the number of common fingerprint bits, and $\left|s_{i} \cup s_{j}\right|$ is the number of union fingerprint bits.

\section{Results and Discussion}

\section{Performance of Predictors}

All molecules in the LIGAND set were used to train the QSAR models, after being transformed into predefined descriptors, including 2048D ECFP6 fingerprints and 19D physicochemical properties. We then tested the performance of these different algorithms with five-fold cross validation and an independent test of which the performances are shown in Fig. 4AB. Here, the dataset was randomly split into five folds in the cross validation, while a temporal split with a cut-off at the year of 2015 was used for the independent test. In the cross validation test, the MT-DNN model achieved the highest value for $\mathrm{R}^{2}$ and the lowest $\mathrm{RMSE}$ value for $\mathrm{A}_{1} \mathrm{AR}$ and $\mathrm{A}_{2 \mathrm{~A}} \mathrm{AR}$, but the RF model had the best performance for hERG based on $\mathrm{R}^{2}$ and RMSE. However, for the independent test the RF model reached the highest $\mathrm{R}^{2}$ and lowest RMSE across the board, although it was worse than the performance in the cross-validation test. A detailed performance overview of the RF model is shown in Fig. 4C-E. Because the generative model might create a large number of novel molecules, which would not be similar to the molecules in the training set, we took the robustness of the predictor into consideration. In this situation the temporal split has been shown to be more robust $[19,35]$. Hence the RF algorithm was chosen for constructing our environment which 
A

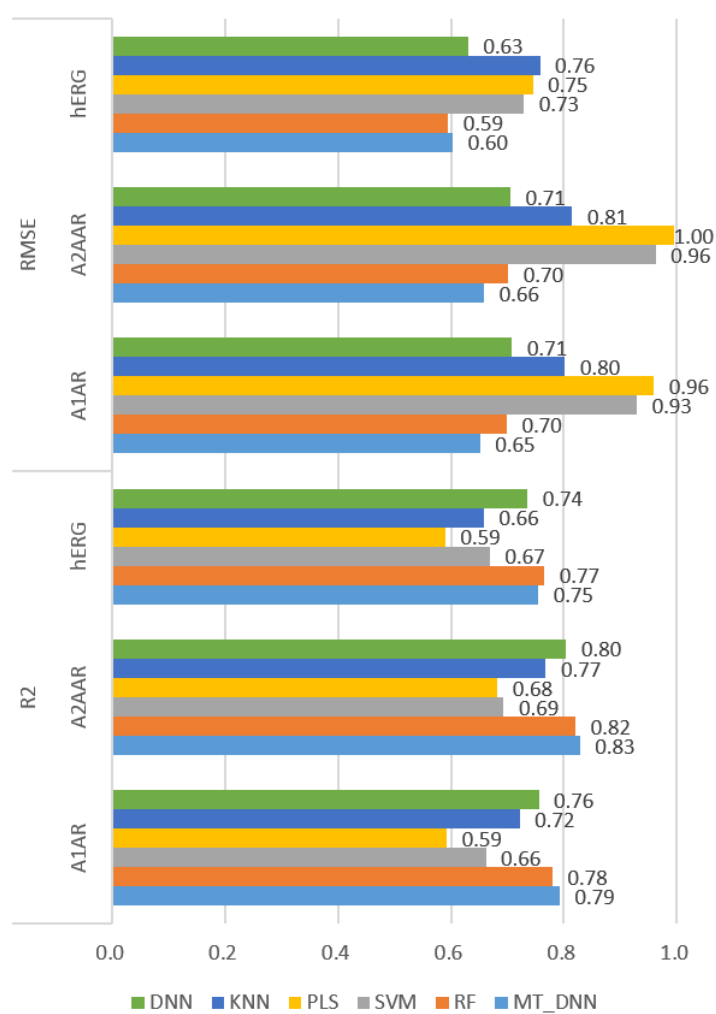

B Independent Test

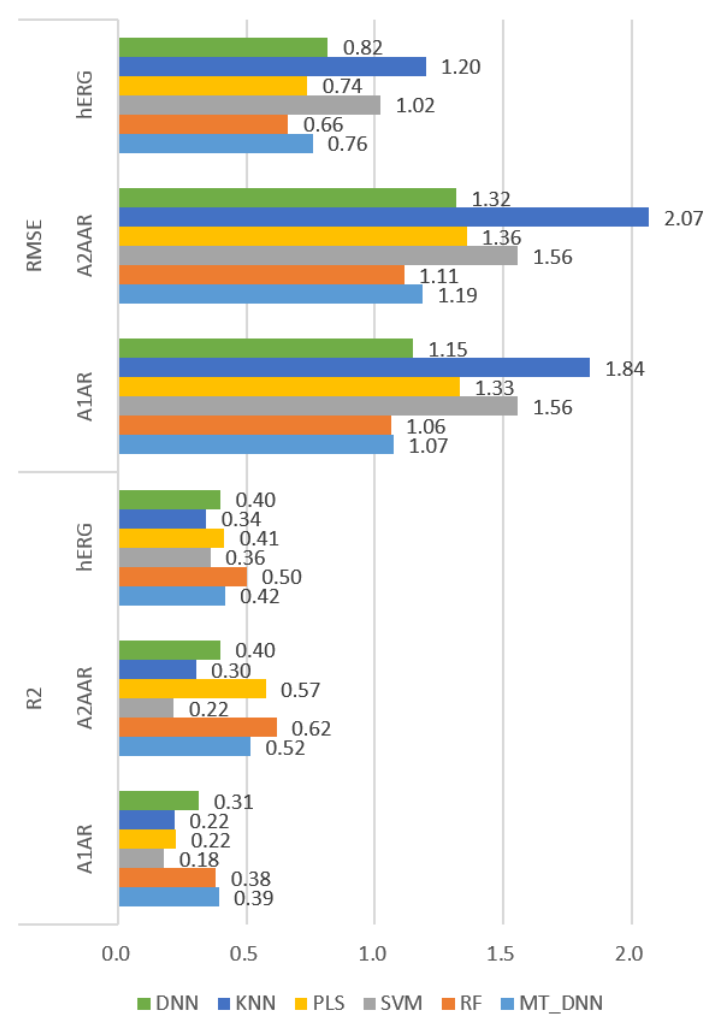

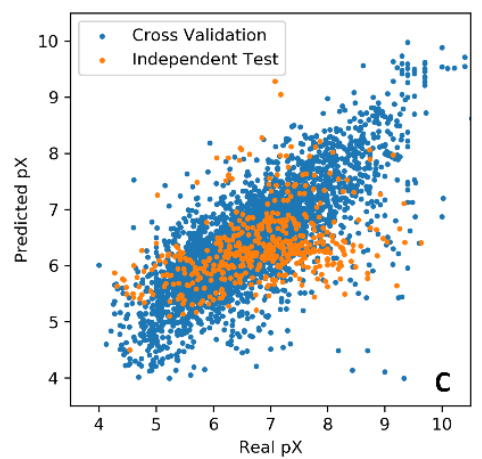
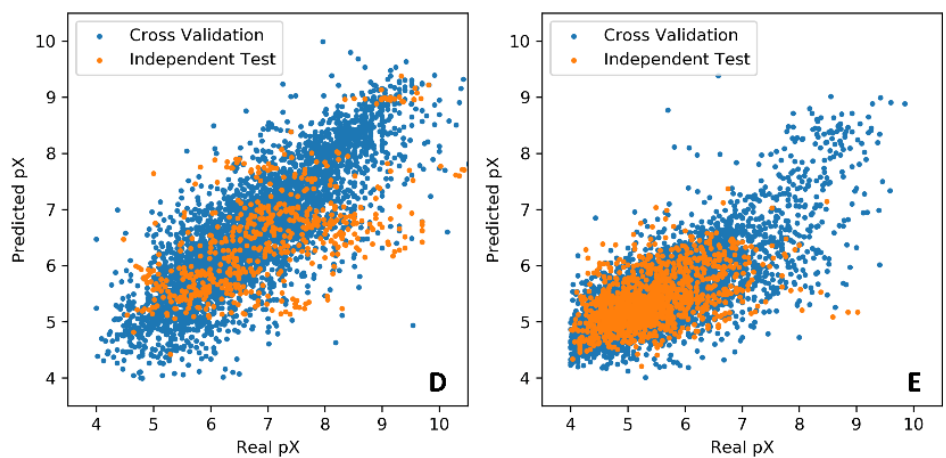

Fig. 4: Performance comparison of different machine learning regression models. In these two

histograms (A-B), the results were obtained based on five-fold cross validation (A) and independent test (B) for the three targets. The $\mathrm{R}^{2}$ and RMSE scores were used to evaluate the performance of different machine learning models including DNN, KNN, PLS, SVM RF and MT-DNN. In the scatter plots (C-E), each point stands for one molecule with its real $\mathrm{pX}(x$-axis) and the predicted pX ( $y$-axis) by the RF model which was chosen as the final predictors for $\mathrm{A}_{1} \mathrm{AR}(\mathrm{C}), \mathrm{A}_{2 \mathrm{~A}} \mathrm{AR}(\mathrm{D})$ and hERG (E) based on five-fold cross validation (blue) and independent test (orange). 
As in our previous work in DrugEx v1, we firstly pre-trained and fine-tuned the generator with the ChEMBL and LIGAND set, respectively. When testing the different types of RNNs, we analyzed the performance of the pre-trained model with 10,000 SMILES generated, and found that LSTM generated more valid SMILES (97.5\%) than GRU (93.1\%) which had been adopted in our previous work. Moreover, for the finetuning process, we split the LIGAND set into two subsets: training set and validation set; the validation set was not involved in parameters updating but it was essential to avoid model overfitting and to improve uniqueness of generated molecules. Subsequently 10,000 SMILES were sampled for performance evaluation. We found that the percentage valid SMILES was $97.9 \%$ for LSTM, larger than GRU with $95.7 \%$ valid SMILES, a slight improvement compared to the pre-trained model. In the end, we employed the LSTM-based pre-trained/fine-tuned models for the following investigation.

We employed the models for two cases (multi-target and target-specific) of multiobjective drug design towards three protein targets. During the training loop of DrugEx $v 2$, the parameter of $\varepsilon$ was set to different values: $10^{-2}, 10^{-3}, 10^{-4}$ and we also tested it without mutation net, i.e. the value of $\varepsilon$ was set to 0 . Generators were trained by using a policy gradient with two different rewarding schemes. After the training process converged, 10,000 SMILES were generated for each model for performance evaluation. The percentage of valid, desired, unique desired SMILES and the diversity were calculated (Table 2). Furthermore, we also compared the chemical space of these generated molecules with known ligands in the LIGAND set. Here, we employed first two components of t-SNE on the ECFP6 descriptors of these molecules to represent the

412 chemical space.

\section{Performance comparisons}

415 We compared the performance of DrugEx v2 with DrugEx $v 1$ and two other DL-based 416 de novo drug design methods: REINVENT [36] and ORGANIC [37]. In order to make 
417 a fair benchmark, we trained these four methods with the same environments to provide 418 the unified predicted bioactivity scores for each of the generated molecules. It should 419 be mentioned that these methods are all SMILES-based RNNs generators but trained 420 under different RL frameworks. Therefore, these generators were constructed with the same RNN structures of and initialized with the same pre-trained/fine-tuned models.

In the WS scheme we did not choose fixed weights for objectives but dynamic values which can be adjusted automatically during the training process. The reason for this is that if the fixed weights should be optimized as the hyperparameters, which would be more time consuming. Moreover, the distribution of scores for each objective was not comparable. If the affinity score was required to be higher, few of the molecules generated by the model with initial state were satisfactory, but if a lower affinity score was required, most of the generated molecules by the pre-trained/fine-tuned model met this need without further training of RL. Therefore, weights were set as dynamic parameters and determined by the ratio between desired and undesired molecules generated by the model at the current training step. This approach ensures that the objectives with lower scores would get more importance than others during the training

The performance of the model with different $\boldsymbol{\varepsilon}$ is shown in Table S2. A higher $\boldsymbol{\varepsilon}$ generates molecules with larger diversity but low desirability compared to a lower $\varepsilon$ in both multi-target and target-specific cases. In addition, an appropriate $\varepsilon$ guarantees the model generates molecules which have a more similar distribution of important substructures with the desired ligands in the LIGAND set. With the WS scheme, the model generates molecules with a high desirability, but the diversity is lower than the

442 desired ligands in the training set. On the contrary, the PF scheme helped the model generate molecules with a larger diversity than the ligands in the training set, but the

444 desirability was not as high as in the WS rewarding scheme. Moreover, the generated molecules in the PF scheme have more similar distribution of substructures to the 446 LIGAND set than in the WS scheme. 
448 Table 2: Comparison of validity, desirability, uniqueness and substructure distributions of 449 SMILES generated by four different methods in the multi-target case with PF and WS 450 rewarding schemes, respectively. For the validity, desirability and uniqueness, the largest data is 451 bold, while for the distribution of substructures, the bold data are labeled as the most closed to the 452 values in the LIGAND set.

\begin{tabular}{|c|c|c|c|c|c|c|c|c|}
\hline $\begin{array}{c}\text { Rewarding } \\
\text { Scheme }\end{array}$ & Dataset & Validity & Desirability & Uniqueness & Diversity & $\begin{array}{c}\text { Purine } \\
\text { Ring }\end{array}$ & $\begin{array}{c}\text { Furan } \\
\text { Ring }\end{array}$ & $\begin{array}{c}\text { Benzene } \\
\text { Ring }\end{array}$ \\
\hline & LIGAND & $100.00 \%$ & $12.40 \%$ & $100.00 \%$ & 0.66 & $21.30 \%$ & $35.44 \%$ & $79.24 \%$ \\
\hline \multirow{4}{*}{$\mathbf{P F}$} & DrugEx $v 1$ & $98.28 \%$ & $43.27 \%$ & $88.96 \%$ & 0.71 & $17.37 \%$ & $41.05 \%$ & $80.95 \%$ \\
\hline & DrugEx v2 & $99.57 \%$ & $80.81 \%$ & $87.29 \%$ & 0.7 & $13.97 \%$ & $32.01 \%$ & $80.26 \%$ \\
\hline & ORGANIC & $98.84 \%$ & $66.01 \%$ & $82.67 \%$ & 0.65 & $17.27 \%$ & $56.38 \%$ & $68.87 \%$ \\
\hline & REINVENT & $99.54 \%$ & $57.43 \%$ & $98.84 \%$ & 0.77 & $0.64 \%$ & $40.38 \%$ & $92.05 \%$ \\
\hline \multirow{4}{*}{ WS } & DrugEx v1 & $97.76 \%$ & $38.44 \%$ & $93.44 \%$ & 0.71 & $10.76 \%$ & $36.42 \%$ & $86.99 \%$ \\
\hline & DrugEx v2 & $99.80 \%$ & $97.45 \%$ & $89.08 \%$ & 0.49 & $3.63 \%$ & $21.06 \%$ & $96.18 \%$ \\
\hline & ORGANIC & $99.08 \%$ & $61.10 \%$ & $77.65 \%$ & 0.68 & $9.08 \%$ & $70.99 \%$ & $83.91 \%$ \\
\hline & REINVENT & $99.54 \%$ & $70.98 \%$ & $99.11 \%$ & 0.71 & $0.04 \%$ & $23.23 \%$ & $96.28 \%$ \\
\hline
\end{tabular}

In the multi-target case, these four methods with different rewarding schemes show similar performance, i.e. the WS scheme can help models improve the desirability while the PF scheme assists models to achieve better diversity and distribution of substructures (Table 2). Here, REINVENT with the PF scheme achieved the largest diversity, whereas DrugEx vl had the most similar substructure distribution to the molecules in the LIGAND set, and DrugEx v2 achieved the best desirability with both PR and WS schemes compared to the three other algorithms. The diversity and distribution of substructures were also most similar to the best results. In addition, in the target-specific case results were similar to the multi-target case, (Table 3), and for the distribution of purine and furan rings, DrugEx v2 surpassed v1 to be most similar to the LIGAND set. When investigating the SA and QED scores, we observed that PF scheme helped all of generated molecules being more drug-like because of higher QED scores than WS scheme in both multi-target case (Fig. 6A-D) and target-specific case 467 (Fig. 6E-H). In comparison of these methods, the molecules generated by REINVENT were supposedly easier to be synthesized and more drug-like than others, but the 

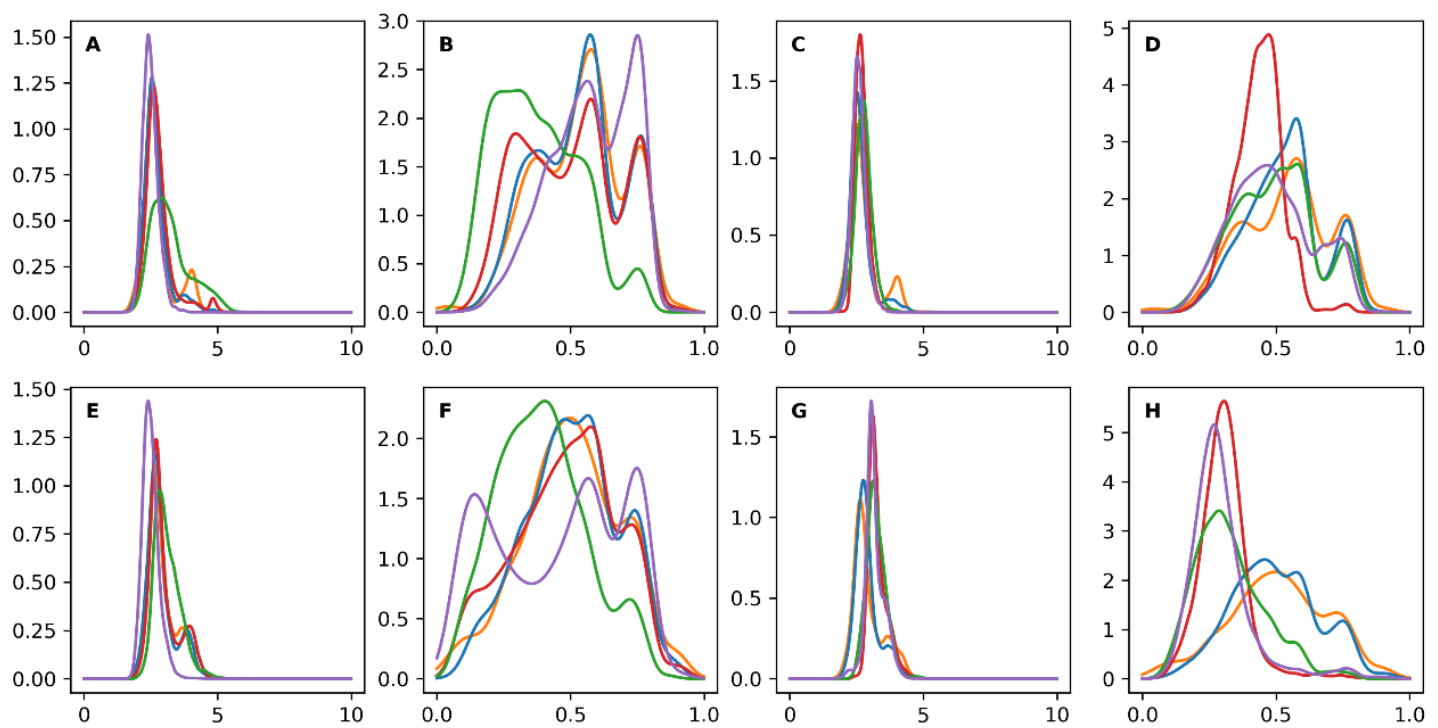

DrugEx v1

DrugEx v

- ORGANIC — REINVENT

479 Fig. 5: the distribution of SA score and QED score of desired ligands in the LIGAND set and 480 of molecules generated by four different methods with PR (A, B, E and F) and WS (C, D, G 
molecules from the LIGAND set were shown as color of orange, and the molecules generated by DrugEx v1, v2, ORGANIC and REINVENT were represented with colors of blue, green, red, and purple, respectively. Overall DrugEx v1 and v2 are better able to emulate the observed distributions in the training set compared to ORGANIC and REINVENT.

With respect to chemical space, we employed t-SNE with the ECFP6 descriptors of all molecules for both multi-target (Fig. 6A-H) and target-specific cases (Fig. 6I-P). In the multi-target case, most of desired ligands in the LIGAND set were distributed in the margin and PR scheme could guide all of the generators to search more regions than WS scheme. In the target-specific case, the desired ligands in the LIGAND set were distributed more dispersed in both of the margin and the center regions. However, PF scheme was not shown the similar results as in the target-specific case to improve the coverage compared with WS scheme except for DrugEx v2. For both of these two cases, only part of the region occupied by desired ligands in the LIGAND set were overlapped with REINVENT and ORGANIC, but almost all of it is covered by DrugEx $v 1$ and $v 2$. Especially, in contrast to WS scheme DrugEx v2 had a significant improvement of chemical space coverage with PF scheme. A possible reason is that the molecules generated by DrugEx $v 1$ and $v 2$ offer a more similar distribution of substructures to desired ligands in the LIGAND set than REINVENT and ORGANIC. 

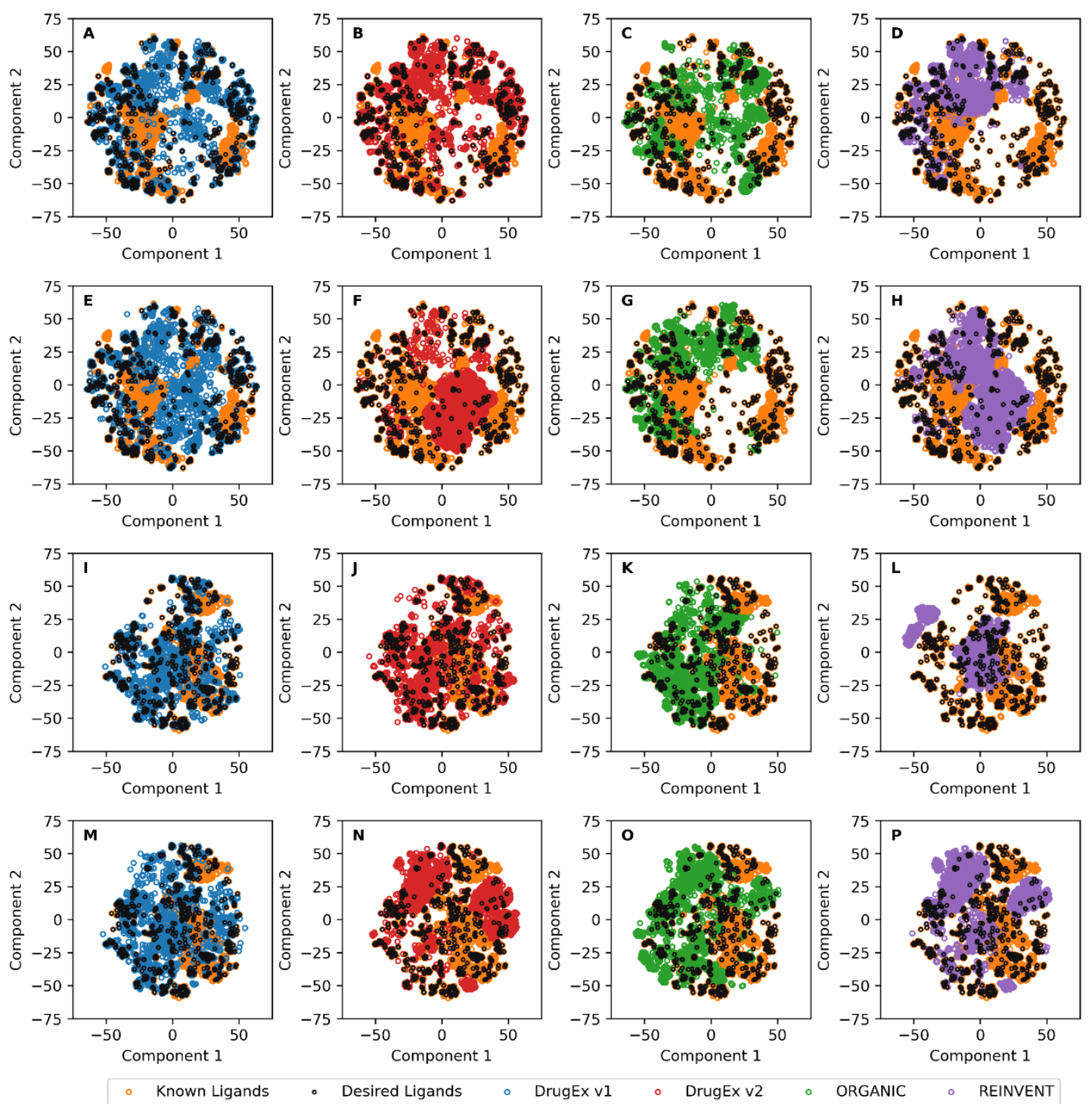

Fig. 6: Comparison of the chemical space of ligands in the LIGAND set (orange for all molecules and black for desired molecules) and of generated molecules by DrugEx v1 (A, E, I, M, blue), v2 (B, F, J, N, red), ORGANIC (C, G, K, O, green) and REINVENT (D, H, L, P, purple), for the multi-target case (A-H) and target specific case (I-P). Chemical space is represented by the first two components in t-SNE with ECFP6 descriptors of molecules. The first and third rows were obtained with PF rewarding scheme, and the second and fourth rows were obtained with WS rewarding scheme. Similar to our previous work it can be seen that DrugEx better covers the whole chemical space of the input data. In particular in the multi-target case with a pareto optimization based scoring function $(\mathrm{E}-\mathrm{H})$ the improved coverage in all sections, including isolated active ligands, becomes clear. 
515 As an example, 16 possible antagonists (without ribose moiety and molecular weight < 516 500) generated by DrugEx v2 with PR scheme were selected as candidates for both 517 multi-target cases and target specific case, respectively. These molecules were ordered 518 by the selectivity which was calculated as the difference of $\mathrm{pXs}$ between two different 519 protein targets. In the multi-target cases (Fig. 7A), because the desired ligands prefer $520 \mathrm{~A}_{1} \mathrm{AR}$ and $\mathrm{A}_{2 \mathrm{~A}} \mathrm{AR}$ to $\mathrm{hERG}$, the row and column is the selectivity of $\mathrm{A}_{2 \mathrm{~A}} \mathrm{AR}$ and $\mathrm{A}_{1} \mathrm{AR}$ 521 against hERG, respectively, while the generated molecules are required to bind only $522 \mathrm{~A}_{2 \mathrm{~A}} \mathrm{AR}$ rather than $\mathrm{A}_{1} \mathrm{AR}$ and $\mathrm{hERG}$ in the target-specific case (Fig. 7B), selectivity of $523 \mathrm{~A}_{2 \mathrm{~A}} \mathrm{AR}$ against $\mathrm{A}_{1} \mathrm{AR}$ and $\mathrm{hERG}$ were represented as the row and column, respectively. 524

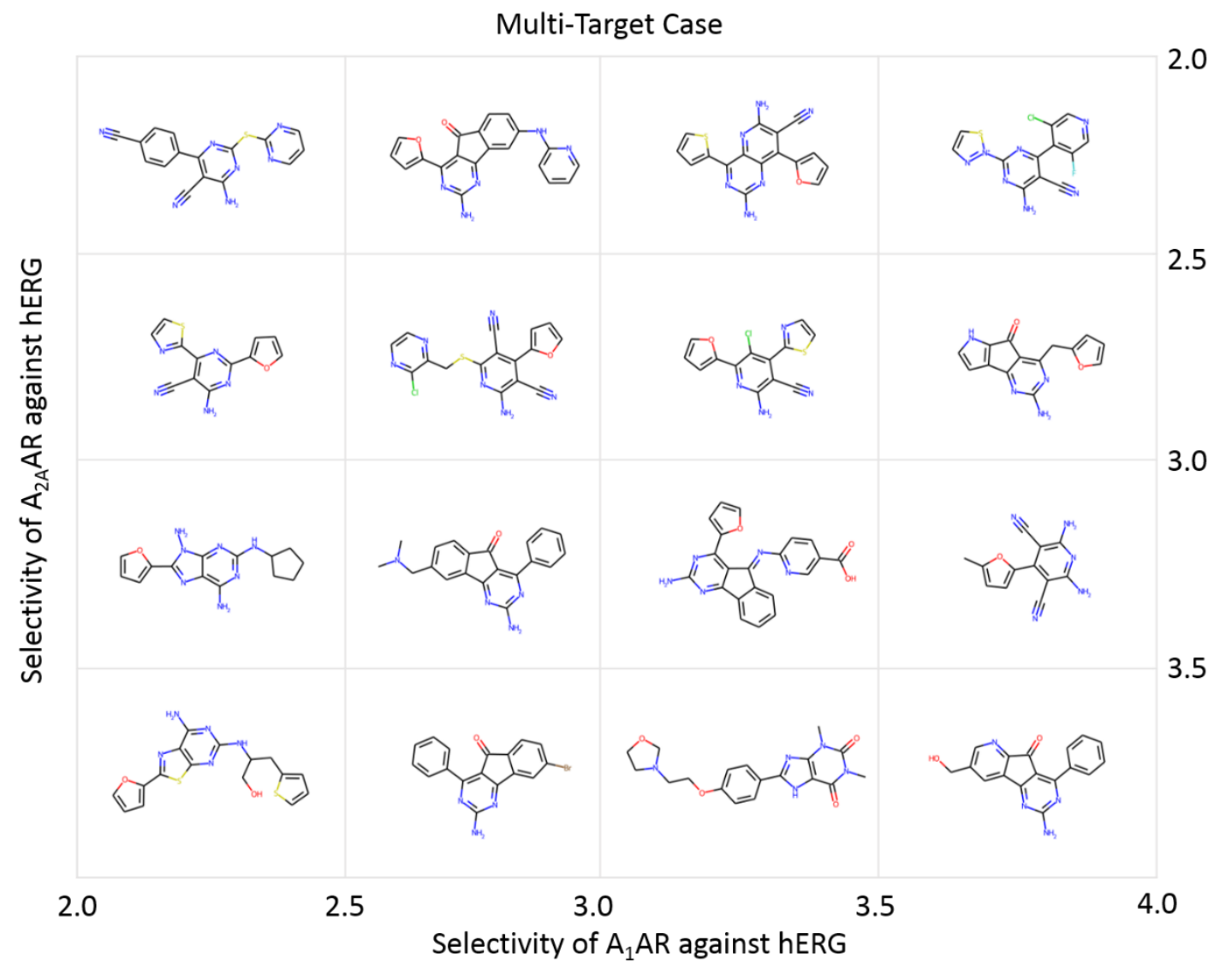




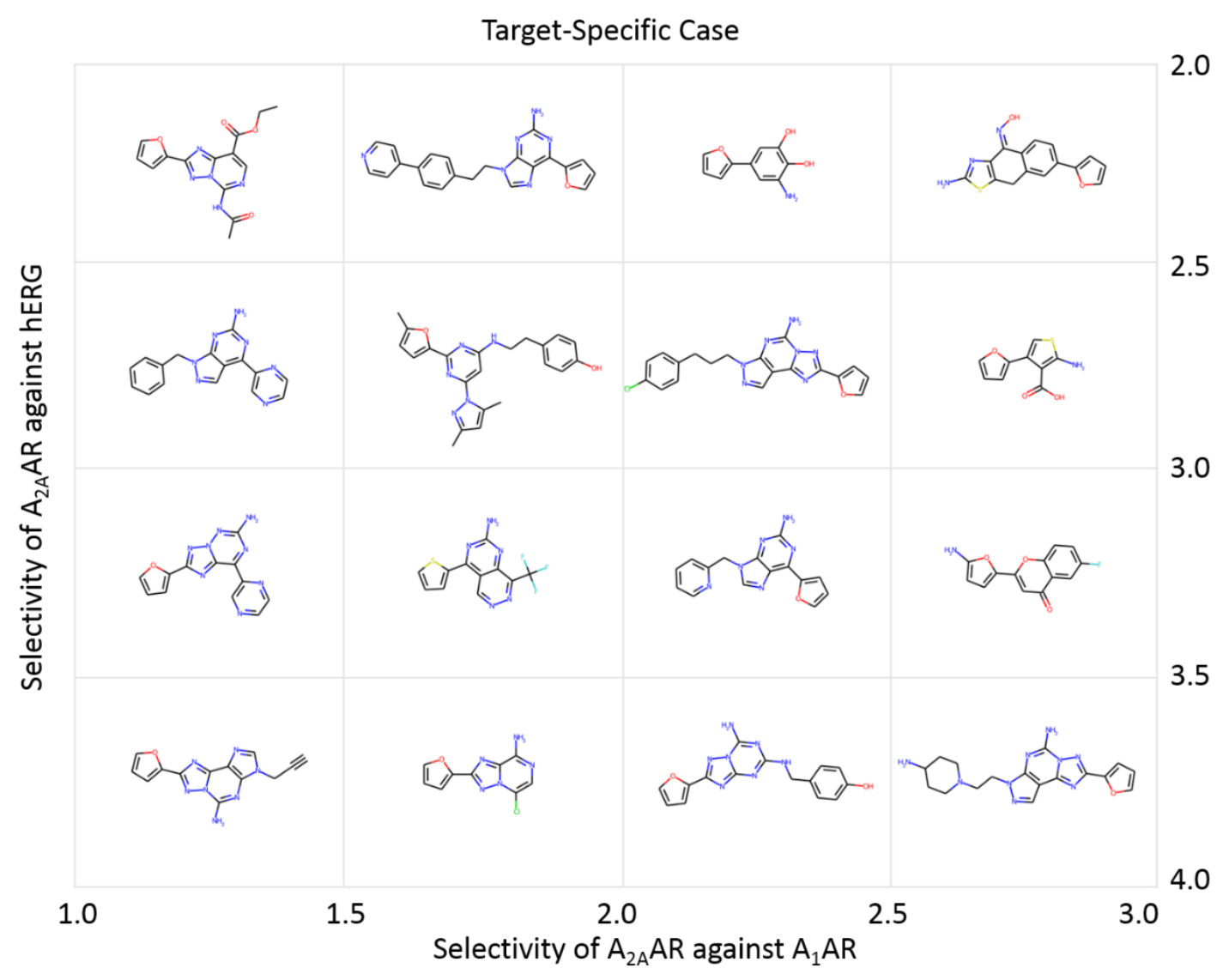

Fig. 7: Some candidate molecules were selected from molecules generated by DrugEx $v 2$ with

PR scheme for both multi-target case and target-specific case. In multi-target case (A), these molecules were ordered by the selectivity of $\mathrm{A}_{1} \mathrm{AR}$ and $\mathrm{A}_{2 \mathrm{~A}} \mathrm{AR}$ against $\mathrm{hERG}$ as $x$-axis and $y$-axis, respectively. In target-specific case (B), these molecules were ordered by the selectivity of $\mathrm{A}_{2 \mathrm{~A}} \mathrm{AR}$ against $\mathrm{A}_{1} \mathrm{AR}$ and hERG as $x$ and $y$-axis, respectively.

In order to prove the effectiveness of our proposed method, we tested it with 20 goaldirected molecule generation tasks on the GuacaMol benchmark platform [38]. These tasks contain different requirements, including similarity, physicochemical properties, isomerism, scaffold matching, etc. The detailed description of these tasks is provided in ref [38] and our results are shown in Table S3. We pre-trained our model with the dataset provided by the GuacaMol platform, in which all molecules from the ChEMBL database are included and similar molecules to the target ligands in the tasks were removed. Then we choose the top 1024 molecules in the training set to fine-tune our model for each task, before reinforcement learning was started. Our method scores the 
542 best in 12 out of 20 tasks compared with the baseline models provided by the GuacaMol

543 platform, leading to an overall second place. Moreover, the performance between the

544 LSTM benchmark method and our methods were similar in these tasks, possibly

545 because they have similar architectures of neural networks. All in all, this benchmark

546 demonstrated that our proposed method has improved generality for drug de novo

547 design tasks. It is worth being mentioned that our method is not effective enough yet

548 for some tasks of contradictory objectives in the narrow chemical space. The main

549 reason is that our method emphasizes to obtain a large number of feasible molecules to

550 occupy the diverse chemical space rather than small number of optimal molecules to

551 achieve the highest score. For example, in the Sitagliptin MPO task, the aim is finding

552 molecules which are dissimilar to sitagliptin but have a similar molecular formula to

553 sitagliptin, and our method was not as good as Graph GA, which is a graph-based 554 genetic algorithm.

555

556 Conclusion and Future Prospects

557 In this work, we proposed a Pareto-based multi-objective learning algorithm for drug

558 de novo design towards multiple targets based on different requirements of affinity 559 scores for multiple targets. We transferred the concept of an evolutionary algorithm 560 (including mutation and crossover operations) into RL to update DrugEx for multi561 objective optimization. In addition, Pareto ranking algorithms were also integrated into 562 our model to handle the contradictory objectives common in drug discovery and enlarge 563 the chemical diversity. In order to prove effectiveness, we tested the performance of 564 DrugEx $v 2$ in both multi-target and target-specific cases. We found that a large 565 percentage of generated SMILES were valid and desired molecules without many 566 duplications. Moreover, the generated molecules were also similar to known ligands 567 and covered almost every corner of the chemical space that known ligands occupy, 568 which could not be repeated by tested competing methods. In future work, we will try 569 the generality of our proposed methods with different molecular representations, such 570 as graphs or fragments [29]. We will also integrate more objectives (e.g. stability, 
571 synthesizability), especially when these objectives are contradictory, such that the 572 model allows user-defined weights for each objective to generate more reliable 573 candidate ligands and better steer the generative process.

\section{Authors' Contributions}

$575 \mathrm{XL}$ and GJPvW conceived the study and performed the experimental work and analysis.

576 KY, APIJ, ME and HWTvV provided feedback and critical input. All authors read, 577 commented on and approved the final manuscript.

578

579 Acknowledgements

580 XL thanks Chinese Scholarship Council (CSC) for funding, GJPvW thanks the Dutch 581 Research Council and Stichting Technologie Wetenschappen (STW) for financial 582 support (STW-Veni \#14410).

584 Competing Interests

585 The authors declare that they have no competing interests 586 


\section{Reference}

589 1. Chaudhari R, Tan Z, Huang B, Zhang S (2017) Computational polypharmacology: a new paradigm for drug discovery. Expert Opin Drug Discov 12 (3):279-291. doi:10.1080/17460441.2017.1280024

2. Giacomini KM, Krauss RM, Roden DM, Eichelbaum M, Hayden MR, Nakamura Y (2007) When good drugs go bad. Nature 446 (7139):975-977. doi:10.1038/446975a

3. Lounkine E, Keiser MJ, Whitebread S, Mikhailov D, Hamon J, Jenkins JL, Lavan P, Weber E, Doak AK, Cote S, Shoichet BK, Urban L (2012) Large-scale prediction and testing of drug activity on side-effect targets. Nature 486 (7403):361-367. doi:10.1038/nature11159

4. Cook D, Brown D, Alexander R, March R, Morgan P, Satterthwaite G, Pangalos MN (2014) Lessons learned from the fate of AstraZeneca's drug pipeline: a five-dimensional framework. Nat Rev Drug Discov 13 (6):419-431. doi:10.1038/nrd4309

5. Siramshetty VB, Nickel J, Omieczynski C, Gohlke BO, Drwal MN, Preissner R (2016) WITHDRAWN--a resource for withdrawn and discontinued drugs. Nucleic Acids Res 44 (D1):D1080-1086. doi:10.1093/nar/gkv1192

6. Hopkins AL (2008) Network pharmacology: the next paradigm in drug discovery. Nat Chem

7. Anighoro A, Bajorath J, Rastelli G (2014) Polypharmacology: challenges and opportunities in 8. van Westen GJ, Wegner JK, Geluykens P, Kwanten L, Vereycken I, Peeters A, IJzerman AP, van Vlijmen HW, Bender A (2011) Which compound to select in lead optimization? Prospectively validated proteochemometric models guide preclinical development. PLoS One 6 (11):e27518. doi:10.1371/journal.pone.0027518

9. Csermely P, Agoston V, Pongor S (2005) The efficiency of multi-target drugs: the network approach might help drug design. Trends Pharmacol Sci 26 (4):178-182. doi:10.1016/j.tips.2005.02.007

10. Fredholm BB (2010) Adenosine receptors as drug targets. Exp Cell Res 316 (8):1284-1288. doi:10.1016/j.yexcr.2010.02.004

11. Fredholm BB, IJzerman AP, Jacobson KA, Linden J, Muller CE (2011) International Union of Basic and Clinical Pharmacology. LXXXI. Nomenclature and classification of adenosine receptors--an update. Pharmacol Rev 63 (1):1-34. doi:10.1124/pr.110.003285

12. Chen JF, Eltzschig HK, Fredholm BB (2013) Adenosine receptors as drug targets--what are the challenges? Nat Rev Drug Discov 12 (4):265-286. doi:10.1038/nrd3955

13. Trudeau MC, Warmke JW, Ganetzky B, Robertson GA (1995) HERG, a human inward rectifier in the voltage-gated potassium channel family. Science 269 (5220):92-95. doi:10.1126/science.7604285

14. Milnes JT, Crociani O, Arcangeli A, Hancox JC, Witchel HJ (2003) Blockade of HERG potassium currents by fluvoxamine: incomplete attenuation by S6 mutations at F656 or Y652. Br J Pharmacol 139 (5):887-898. doi:10.1038/sj.bjp.0705335

15. Sanguinetti MC, Tristani-Firouzi M (2006) hERG potassium channels and cardiac arrhythmia. Nature 440 (7083):463-469. doi:10.1038/nature04710 doi:10.1038/nature14539 
17. Chen H, Engkvist O, Wang Y, Olivecrona M, Blaschke T (2018) The rise of deep learning in

19. Lenselink EB, Ten Dijke N, Bongers B, Papadatos G, van Vlijmen HWT, Kowalczyk W,

20. Liu X, Ye K, van Vlijmen HWT, IJzerman AP, van Westen GJP (2019) An exploration strategy IJzerman AP, van Westen GJP (2017) Beyond the hype: deep neural networks outperform established methods using a ChEMBL bioactivity benchmark set. Journal of cheminformatics 9 (1):45. doi:10.1186/s13321-017-0232-0

21. RDKit: Open-Source Cheminformatics Software. http://www.rdkit.org.

22. Rogers D, Hahn M (2010) Extended-connectivity fingerprints. Journal of chemical information

648 23. Scikit-Learn: machine learning in Python. http://www.scikit-learn.org/.

649 24. PyTorch. https://pytorch.org/.

650 25. Kingma DP, Ba J (2014) Adam: A Method for Stochastic Optimization. arXiv:1412.6980

651 26. Chung J, Gulcehre C, Cho K, Bengio Y (2014) Empirical Evaluation of Gated Recurrent Neural

27. Deb K, Agrawal S, Pratap A, Meyarivan T A Fast Elitist Non-dominated Sorting Genetic

28. Emmerich MTM, Deutz AH (2018) A tutorial on multiobjective optimization: fundamentals

29. Liu X, IJzerman AP, van Westen GJP (2021) Computational Approaches for De Novo Drug

30. Lameijer EW, Kok JN, Back T, IJzerman AP (2006) The molecule evoluator. An interactive

31. van der Horst E, Marques-Gallego P, Mulder-Krieger T, van Veldhoven J, Kruisselbrink J, Statistics 1 (2):95-103. doi:10.1007/BF02426650 Application of portfolio optimization to drug discovery. Information Sciences 475:29-43. 
doi:https://doi.org/10.1016/j.ins.2018.09.049

35. Sheridan RP (2013) Time-split cross-validation as a method for estimating the goodness of prospective prediction. Journal of chemical information and modeling 53 (4):783-790. doi: $10.1021 / \mathrm{ci} 400084 \mathrm{k}$

36. Olivecrona M, Blaschke T, Engkvist O, Chen H (2017) Molecular de-novo design through deep reinforcement learning. Journal of cheminformatics 9 (1):48. doi:10.1186/s13321-017-0235-x

37. Benjamin S-L, Carlos O, Gabriel L. G, Alan A-G (2017) Optimizing distributions over molecular space. An Objective-Reinforced Generative Adversarial Network for Inverse-design Chemistry (ORGANIC). doi:10.26434/chemrxiv.5309668.v3

38. Brown N, Fiscato M, Segler MHS, Vaucher AC (2019) GuacaMol: Benchmarking Models for 685 de Novo Molecular Design. Journal of chemical information and modeling 59 (3):1096-1108. doi:10.1021/acs.jcim.8b00839

687

688 
Table S1: All tokens in vocabulary for SMILES sequence construction with RNN model.

\begin{tabular}{|c|c|c|c|c|c|c|c|c|c|c|}
\hline \multicolumn{7}{|c|}{ Atoms } & \multirow{3}{*}{$\begin{array}{c}\text { Bonds } \\
-- \\
-\end{array}$} & \multicolumn{3}{|c|}{ Controls } \\
\hline \multicolumn{5}{|c|}{ Common Atoms } & \multicolumn{2}{|c|}{ Aromatic Atoms } & & Rings & Branchs & On-Off \\
\hline B & {$[\mathrm{Ag}-3]$} & {$[\mathrm{CH}-]$} & {$[\mathrm{N}]$} & [SH2] & {$[b-]$} & {$[\mathrm{se}+]$} & & 1 & ( & GO \\
\hline $\mathrm{C}$ & {$[\mathrm{As}+]$} & [CH2] & {$[\mathrm{O}+]$} & {$[\mathrm{SH}]$} & {$[\mathrm{c}+]$} & [se] & $=$ & 2 & ) & EOS \\
\hline $\mathrm{F}$ & {$[\mathrm{As}]$} & {$[\mathrm{CH}]$} & {$[\mathrm{O}-]$} & {$[\mathrm{Se}+]$} & [c-] & {$[$ te +$]$} & $\#$ & 3 & & \\
\hline I & {$[\mathrm{B}-]$} & {$[\mathrm{I}+]$} & {$[\mathrm{OH}+]$} & {$[\mathrm{SeH}]$} & {$[\mathrm{cH}-]$} & [te] & & 4 & & \\
\hline $\mathrm{L}$ & {$[\mathrm{BH}-]$} & [IH2] & {$[\mathrm{O}]$} & {$[\mathrm{Se}]$} & {$[\mathrm{n}+]$} & $\mathrm{b}$ & & 5 & & \\
\hline $\mathrm{N}$ & [BH2-] & {$[\mathrm{N}+]$} & {$[\mathrm{P}+]$} & {$[\mathrm{SiH} 2]$} & {$[\mathrm{n}-]$} & $\mathrm{c}$ & & 6 & & \\
\hline $\mathrm{O}$ & [BH3-] & {$[\mathrm{N}-]$} & {$[\mathrm{PH}]$} & {$[\mathrm{SiH}]$} & {$[\mathrm{nH}+]$} & $\mathrm{n}$ & & 7 & & \\
\hline $\mathrm{P}$ & {$[\mathrm{B}]$} & {$[\mathrm{NH}+]$} & {$[\mathrm{S}+]$} & {$[\mathrm{Si}]$} & {$[\mathrm{nH}]$} & o & & 8 & & \\
\hline $\mathrm{R}$ & {$[\mathrm{C}+]$} & {$[\mathrm{NH}-]$} & {$[\mathrm{S}-]$} & {$[\mathrm{Te}]$} & {$[0+]$} & $\mathrm{p}$ & & 9 & & \\
\hline S & [C- $]$ & {$[\mathrm{NH} 2+]$} & {$[\mathrm{SH}+]$} & & {$[\mathrm{s}+]$} & $\mathrm{s}$ & & & & \\
\hline
\end{tabular}

690 Considering that the sterochemical information of molecules and ionic bonds were ignored, we removed 691 the “@”, “””, “/”, “.”. 
693 SMILES generated by DrugEx $v 2$ with different $\varepsilon$ in the multi-target and target-specific cases

694 by using PF and WS rewarding schemes, respectively. For the validity, desirability and 695 uniqueness, the largest data is bold, while for the distribution of substructures, the bold data are 696 labeled as the most closed to the values in the LIGAND set.

\begin{tabular}{|c|c|c|c|c|c|c|c|c|c|}
\hline Case & $\begin{array}{l}\text { Reward } \\
\text { Scheme }\end{array}$ & $\begin{array}{c}\text { Dataset } \\
\qquad / \varepsilon\end{array}$ & Validity & Desirability & Uniqueness & Diversity & $\begin{array}{c}\text { Purine } \\
\text { Ring }\end{array}$ & $\begin{array}{c}\text { Furan } \\
\text { Ring }\end{array}$ & $\begin{array}{c}\text { Benzene } \\
\text { Ring }\end{array}$ \\
\hline \multirow{9}{*}{$\begin{array}{l}\text { Multi- } \\
\text { Target } \\
\text { Case }\end{array}$} & & LIGAND & $100.00 \%$ & $14.63 \%$ & $100.00 \%$ & 0.67 & $21.30 \%$ & $35.44 \%$ & $79.24 \%$ \\
\hline & \multirow{4}{*}{$\mathrm{PF}$} & $10^{-2}$ & $99.39 \%$ & $71.37 \%$ & $\mathbf{9 0 . 4 7 \%}$ & 0.72 & $12.39 \%$ & $34.69 \%$ & $82.05 \%$ \\
\hline & & $10^{-3}$ & $99.57 \%$ & $80.81 \%$ & $88.96 \%$ & 0.71 & $13.97 \%$ & $32.01 \%$ & $80.26 \%$ \\
\hline & & $10^{-4}$ & $99.72 \%$ & $83.86 \%$ & $87.19 \%$ & 0.71 & $12.45 \%$ & $30.58 \%$ & $84.04 \%$ \\
\hline & & 0 & $99.47 \%$ & $73.76 \%$ & $84.41 \%$ & 0.70 & $13.35 \%$ & $35.71 \%$ & $81.89 \%$ \\
\hline & \multirow{4}{*}{ WS } & $10^{-2}$ & $99.54 \%$ & $87.56 \%$ & $93.08 \%$ & 0.60 & $9.66 \%$ & $28.83 \%$ & $92.19 \%$ \\
\hline & & $10^{-3}$ & $99.80 \%$ & $97.45 \%$ & $93.44 \%$ & 0.49 & $3.63 \%$ & $21.06 \%$ & $96.18 \%$ \\
\hline & & $10^{-4}$ & $99.79 \%$ & $98.15 \%$ & $93.56 \%$ & 0.53 & $2.89 \%$ & $24.95 \%$ & $91.46 \%$ \\
\hline & & 0 & $99.78 \%$ & $98.00 \%$ & $90.19 \%$ & 0.49 & $5.02 \%$ & $16.45 \%$ & $96.77 \%$ \\
\hline \multirow{4}{*}{ Target- } & \multirow{5}{*}{$\mathrm{PF}$} & LIGAND & $100.00 \%$ & $12.40 \%$ & $100.00 \%$ & 0.66 & $28.27 \%$ & $50.61 \%$ & $71.84 \%$ \\
\hline & & $10^{-2}$ & $99.48 \%$ & $88.76 \%$ & $91.98 \%$ & 0.77 & $18.31 \%$ & $47.50 \%$ & $68.95 \%$ \\
\hline & & $10^{-3}$ & $99.53 \%$ & $89.49 \%$ & $87.32 \%$ & 0.72 & $23.73 \%$ & $56.23 \%$ & $67.40 \%$ \\
\hline & & $10^{-4}$ & $99.55 \%$ & $91.84 \%$ & $88.31 \%$ & 0.74 & $26.86 \%$ & $39.68 \%$ & $74.36 \%$ \\
\hline Specific & & 0 & $99.54 \%$ & $91.47 \%$ & $88.94 \%$ & 0.75 & $22.95 \%$ & $43.08 \%$ & $71.50 \%$ \\
\hline \multirow[t]{4}{*}{ Case } & \multirow{4}{*}{ WS } & $10^{-2}$ & $99.16 \%$ & $86.45 \%$ & $93.97 \%$ & 0.42 & $42.84 \%$ & $97.26 \%$ & $72.45 \%$ \\
\hline & & $10^{-3}$ & $99.62 \%$ & $97.86 \%$ & $95.89 \%$ & 0.31 & $60.81 \%$ & $98.56 \%$ & $51.87 \%$ \\
\hline & & $10^{-4}$ & $99.67 \%$ & $96.82 \%$ & $94.56 \%$ & 0.34 & $55.14 \%$ & $93.69 \%$ & $45.40 \%$ \\
\hline & & 0 & $99.33 \%$ & $96.28 \%$ & $92.60 \%$ & 0.35 & $42.86 \%$ & $98.34 \%$ & $63.47 \%$ \\
\hline
\end{tabular}


699 Table S3: Results of the Goal-Directed tasks for our proposed method DrugEx v2 and other baseline

700 models on GuacaMol Benchmark. GucacaMol platform contains 20 tasks with different requirements,

701 including smilarity, physicochemical properties, isomerism, scaffold matching, etc.. The results for

702 baseline models were cited from ref [38]. The bold data are shown as the best result for each task

703 achieved by different methods.

\begin{tabular}{|c|c|c|c|c|c|c|}
\hline \multirow[t]{2}{*}{ Benchmark } & Best of & SMILES & Graph & Graph GA & SMILES & DrugEx \\
\hline & Dataset & GA & MCTS & & LSTM & v2 \\
\hline Celecoxib rediscovery & 0.505 & 0.732 & 0.355 & 1 & 1 & 1 \\
\hline Troglitazone rediscovery & 0.419 & 0.515 & 0.311 & 1 & 1 & 1 \\
\hline Thiothixene rediscovery & 0.456 & 0.598 & 0.311 & 1 & 1 & 1 \\
\hline Aripiprazole similarity & 0.595 & 0.834 & 0.38 & 1 & 1 & 1 \\
\hline Albuterol similarity & 0.719 & 0.907 & 0.749 & 1 & 1 & 1 \\
\hline Mestranol similarity & 0.629 & 0.79 & 0.402 & 1 & 1 & 1 \\
\hline $\mathrm{C} 11 \mathrm{H} 24$ & 0.684 & 0.829 & 0.41 & 0.971 & 0.993 & 0.993 \\
\hline $\mathrm{C} 9 \mathrm{H} 10 \mathrm{~N} 2 \mathrm{O} 2 \mathrm{PF} 2 \mathrm{Cl}$ & 0.747 & 0.889 & 0.631 & 0.982 & 0.879 & 1 \\
\hline Median molecules 1 & 0.334 & 0.334 & 0.225 & 0.406 & 0.438 & 0.418 \\
\hline Median molecules 2 & 0.351 & 0.38 & 0.17 & 0.432 & 0.422 & 0.435 \\
\hline Osimertinib MPO & 0.839 & 0.886 & 0.784 & 0.953 & 0.907 & 0.967 \\
\hline Fexofenadine MPO & 0.817 & 0.931 & 0.695 & 0.998 & 0.959 & 0.942 \\
\hline Ranolazine MPO & 0.792 & 0.881 & 0.616 & 0.92 & 0.855 & 0.909 \\
\hline Perindopril MPO & 0.575 & 0.661 & 0.385 & 0.792 & 0.808 & 0.812 \\
\hline Amlodipine MPO & 0.696 & 0.722 & 0.533 & 0.894 & 0.894 & 0.898 \\
\hline Sitagliptin MPO & 0.509 & 0.689 & 0.458 & 0.891 & 0.545 & 0.517 \\
\hline Zaleplon MPO & 0.547 & 0.413 & 0.488 & 0.754 & 0.669 & 0.693 \\
\hline Valsartan SMARTS & 0.259 & 0.552 & 0.04 & 0.99 & 0.978 & 0.978 \\
\hline Scaffold Hop & 0.933 & 0.97 & 0.59 & 1 & 0.996 & 0.989 \\
\hline Deco Hop & 0.738 & 0.885 & 0.478 & 1 & 0.998 & 0.986 \\
\hline Total & 12.144 & 14.398 & 9.011 & 17.983 & 17.341 & 17.537 \\
\hline
\end{tabular}



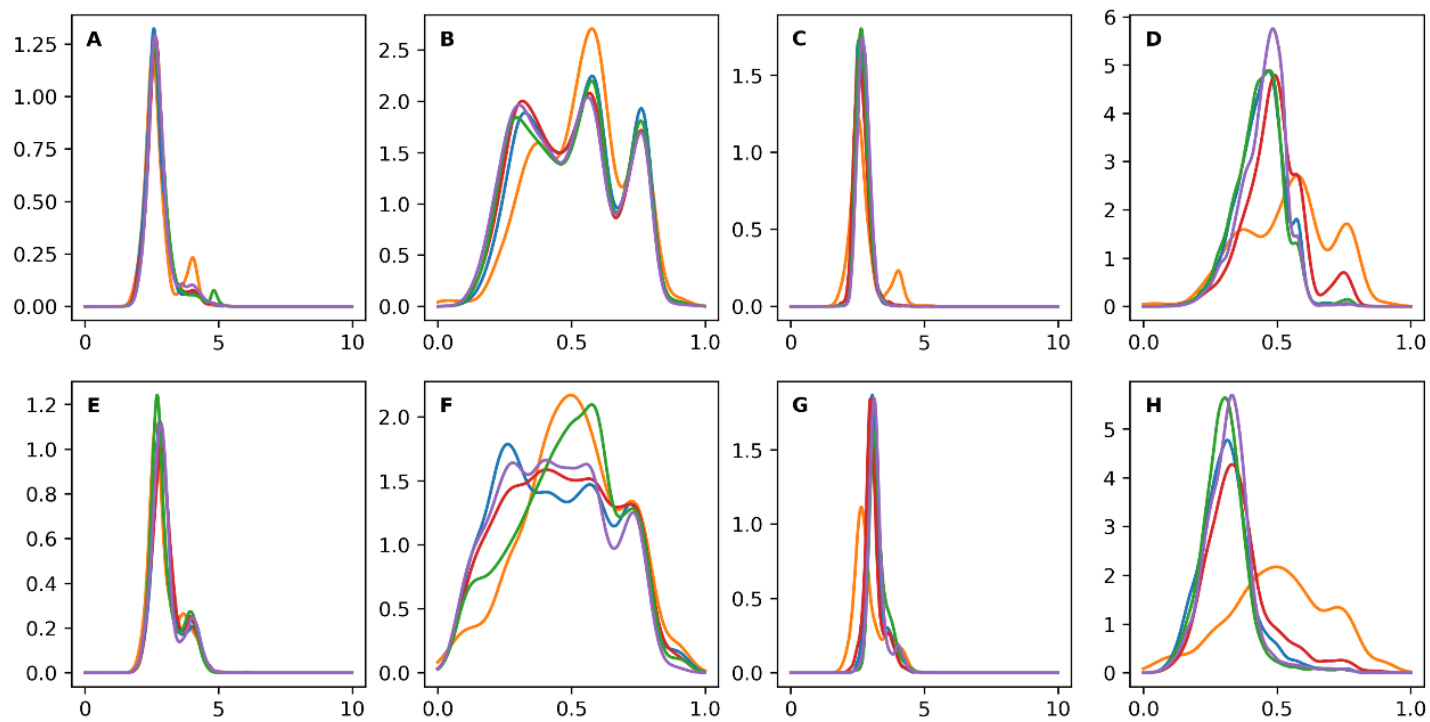

$=0 \mathrm{e}+00$

$\varepsilon=1 \mathrm{e}-04$

Fig. S1: the distribution of SA score and QED score of desired ligand in the LIGAND set and molecules generated by DrugEx v2 with different $\varepsilon$ in the multi-target case (A-D) and targetspecific case (E-H) by using PR (A, B, E and F) and WS (C, D, G and H) rewarding schemes. 